\title{
Simple Strategy for Rapid and Sensitive Detection of 2019 novel coronavirus Based on Antibody
}

\section{Xinjun Hu ( $\square$ hxj5129@163.com )}

Department of Infectious Diseases, The First Affiliated Hospital and College of Clinical Medicine of Henan University of Science and Technology

\section{Huiyang Deng}

Department of Infectious Diseases, The First Affiliated Hospital and College of Clinical Medicine of Henan University of Science and Technology

\section{Yibing Shang}

Department of Infectious Diseases, The First Affiliated Hospital and College of Clinical Medicine of Henan University of Science and Technology

\section{Mengnan Fan}

Department of Infectious Diseases, The First Affiliated Hospital and College of Clinical Medicine of Henan University of Science and Technology

\section{Feng Yue}

Department of Infectious Diseases, The First Affiliated Hospital and College of Clinical Medicine of Henan University of Science and Technology

\section{Short Report}

Keywords: 2019-nCoV, RT-PCR, Antibody

Posted Date: March 6th, 2020

DOl: https://doi.org/10.21203/rs.3.rs-16761/v1

License: (a) (1) This work is licensed under a Creative Commons Attribution 4.0 International License. Read Full License 


\section{Abstract}

Objectives: Since December 2019, acute respiratory disease due to 2019 novel coronavirus emerged in Wuhan city and rapidly spread throughout China. Real-time RT-PCR is widely deployed in diagnostic virology. However, the positive detection rates of RT-PCR are only $30 \%$ to $50 \%$. Therefore, we propose a simple strategy for rapidly and sensitively detecting the $\operatorname{lgM} / \mathrm{lgG}$ antibody against 2019-nCoV using a colloidal gold-based immunochromatographic strip test.

Methods A total of 41 clinically 2019 -nCoV suspected cases (23 males and 18 females) were enrolled. The sensitivity of colloidal gold-based immunochromatographic strip test and of RT-PCR were compared and evaluated. McNemar's test was used to compare the detection rate of both assays $(P<0.05)$.

Results: The Antibody was detected in $63.4 \%$ (26/41) of blood specimens using the assay. In contrast, the 2019-nCoV was detected in 46.3\% (19/41) of nasal and pharyngeal swab specimens using the RT-PCR assays. The detection rate obtained by this assay was markedly higher than that obtained by the RT-PCR assays $(P=0.039)$

Conclusion: This detection assay exhibits a higher detection sensitivity than RT-PCR. More important, the assay shows the benefits of easy operation and setup. We believe that the sensitive and time-saving approach may be used as an auxiliary diagnostic tool for 2019-nCoV detection and virus screening and confirmation.

\section{Introduction}

According to the World Health Organization (WHO), the WHO China Country Office was informed of cases of pneumonia of unknown aetiology in Wuhan City, Hubei Province, on 31 December 2019¹. Highthroughput sequencing has revealed a novel betacoronavirus that is currently named 2019 novel coronavirus (2019-nCoV) ${ }^{2}$. Evidence pointing to the person-to-person transmission in hospital and family settings has been accumulating ${ }^{3-6}$. Lots of patients who are infected with the $2019-\mathrm{nCoV}$ progress to pneumonia or acute respiratory distress syndrome ${ }^{7}$. As of February 27 th, 2020खa total of 78,630 laboratory-confirmed cases of human infection with 2019-nCoV in China, and among them, 2009 ended in death ${ }^{8}$. A confirmed case with 2019-nCoV ARD was defined as a positive result to high-throughput sequencing or real-time reverse-transcriptase polymerase-chain-reaction (RT-PCR) assay for nasal and pharyngeal swab specimens ${ }^{1}$. However, high-throughput sequencing is time-consuming and complex. Although RT-PCR is relatively time-saving, it still needs several hours. At present, the positive detection rates of RT-PCR are only $30 \%$ to $50 \%$. Additionally, high cost and the need of well-trained technical staff further limit the technique ${ }^{9-11}$. Therefore, there is an urgent demand for developing a simple, costeffective, sensitive, and specific assay to rapidly diagnose infections with the 2019-nCoV. We propose a simple strategy for rapidly and sensitively detecting the $\operatorname{lgM} / \mathrm{lgG}$ antibody against 2019-nCoV using a colloidal gold-based immunochromatographic strip test. Most importantly, the assay time took less than 
5 min. In this study, the sensitivity of colloidal gold-based immunochromatographic strip test and of RTPCR were compared and evaluated.

\section{Patients And Methods}

Study Population. A total of 41 clinically 2019 -nCoV suspected cases (23 males and 18 females) which from the First Affiliated Hospital of Henan University of Science and Technology were enrolled. Suspected cases were identified as having fever, or respiratory symptoms, or radiologic abnormalities on computed tomography, and a history of exposure to wildlife in Wuhan seafood market, a travel history or contact with a confirmed case within 2 weeks. Written informed consent forms were provided and signed by the study participants, and the study procedures were conducted in agreement with ethics committee regulations. The study was approved by the Institutional Ethics Committee of the First Affiliated Hospital of Henan University of Science and Technology.

Procedures. Laboratory confirmation of the 2019-nCoV was achieved through the concerted efforts of Luoyang Center for Disease Prevention and Control (CDC). The RT-PCR assay for nasal and pharyngeal swab specimens was conducted in accordance with the protocol established by the World Health Organization. The detection of IgM/lgG antibody was performed using the colloidal gold-based immunochromatographic strip test kit(Zhu Hai Livzon Diagnostics INC, Zhuhai, China). Briefly, the assay is performed by adding $10 \mu \mathrm{l}$ of serum/plasma or $20 \mu \mathrm{l}$ of whole blood, and 2 drops of the assay buffer. Reacting bands were read after 1-5 min. The final results were agreed upon by 2 investigators(Fig.1).

\section{Statistical analysis}

Statistical analyses were performed using IBM SPSS Statistics Version 22.0. McNemar's test was used to compare the detection rates of the assay and RT-PCR. Differences were considered statistically significant when $P<0.05$.

\section{Results}

The Antibody was detected in $63.4 \%$ (26/41) of blood specimens using this assay. Among them, the IgM was detected in $29.3 \%$ (12/41) of blood specimens. The IgG was detected in $46.3 \%$ (19/41) of blood specimens. In contrast, the 2019-nCoV was detected in $46.3 \%$ (19/41) of nasal and pharyngeal swab specimens using the RT-PCR assays. The detection rate obtained by the assay was markedly higher than that obtained by the RT-PCR assays $(P=0.039)$ (Table 1$)$.

Table 1. Sensitivity of this assay 


\begin{tabular}{ccccc}
\hline & & \multicolumn{2}{c}{ RT-PCR } & \\
& & & & Total \\
\hline & Positive & Negative & \\
\hline The assay & Positive & 18 & 8 & 26 \\
\hline Total & Negative & 1 & 14 & 15 \\
\hline & & 19 & 22 & 41 \\
\hline
\end{tabular}

\section{Discussion}

Rapid and accurate diagnosis of respiratory viruses can help in epidemiologic monitoring, along with taking effective prevention steps and implementing appropriate antiviral therapies. In the present case of 2019-nCoV, RT-PCR is widely deployed in diagnostic virology. In this study, the detection assay exhibits a higher detection sensitivity than RT-PCR. More important, this assay shows the benefits of easy operation and setup. Thus, We believe that the sensitive and time-saving approach may be used as an auxiliary diagnostic tool for 2019-nCoV detection and virus screening and confirmation. Nevertheless, further systematic investigations on clinical specimens collected from 2019-nCoV-infected patients at different post-onset time points will be needed.

\section{Declarations}

\section{Conflict of interest}

The authors declare that they have no confict of interest.

\section{Acknowledgment:}

We thank the Luoyang Center for Disease Prevention and Control, Beike Biotechnology and Zhu Hai Livzon Diagnostics INC. This study was supported by the National Natural Science Foundation of China (81600512) and Luoyang Science and Technology Funds.

\section{Author Contributions}

X.H, F. Y. conceived and designed the experiments. X.H, H. D.and Y. S. performed the experiments. X.H, M. F.analysed the data. X. H.drew the all fgures and wrote the paper.

\section{Figures}




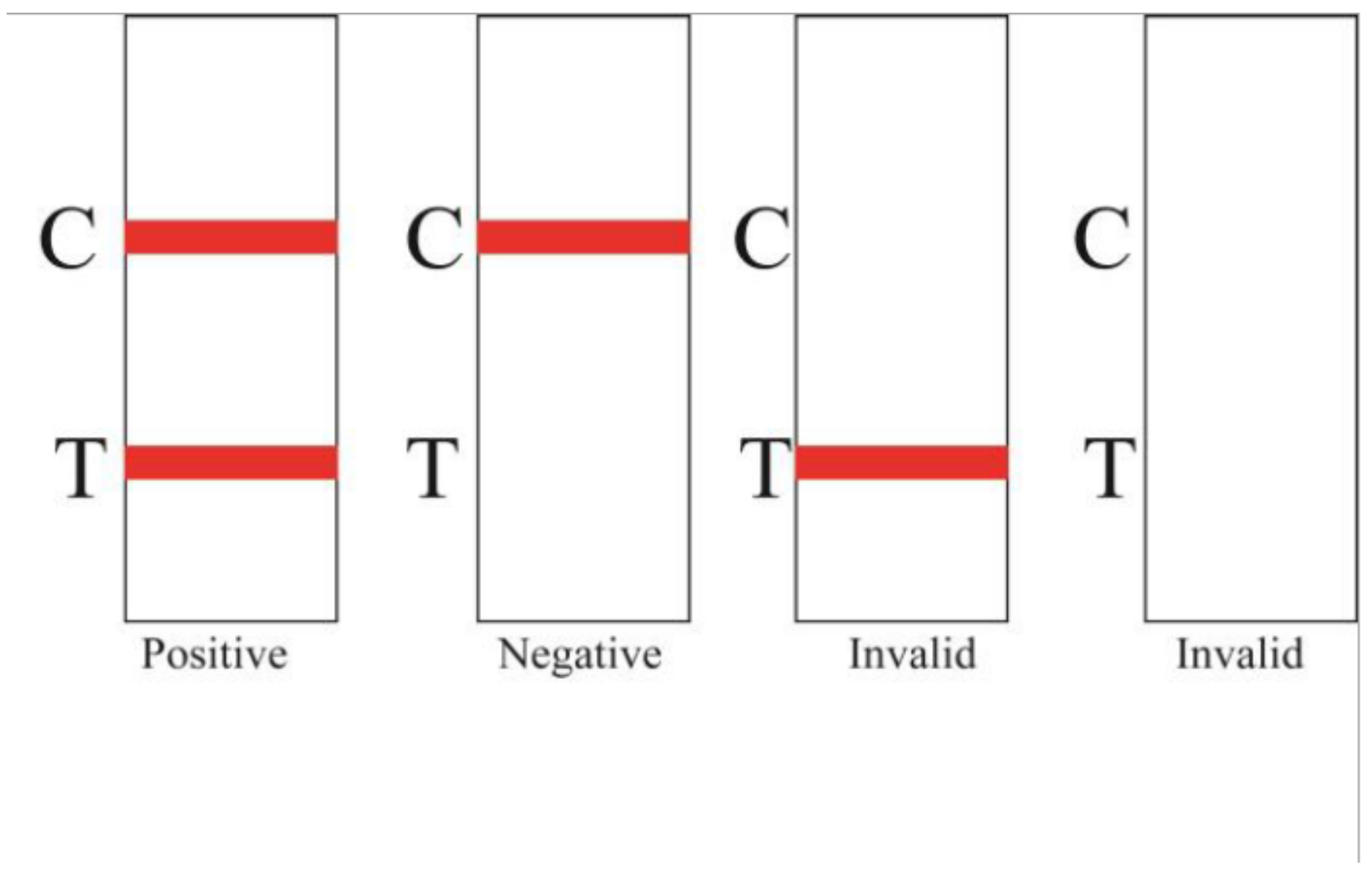

Figure 1

The results of the assay. The procedure does not require any specific technic, can be tested easily and rapidly, and showed clear results. 